\title{
Factors associated with diagnosis and treatment of osteoporosis in older adults
}

\author{
S. Nayak • M. S. Roberts • S. L. Greenspan
}

Received: 17 October 2008 / Accepted: 10 December 2008 / Published online: 17 January 2009

(C) The Author(s) 2009. This article is published with open access at Springerlink.com

\begin{abstract}
Summary Osteoporosis is often undiagnosed and untreated. We surveyed 1,830 adults and identified factors associated with osteoporosis diagnosis and treatment. Individuals with several risk factors, including older age, were not more likely to be diagnosed or treated. Measures should be taken to improve osteoporosis identification and treatment in high-risk patients.

Introduction We aimed to identify patient characteristics associated with osteoporosis diagnosis and treatment.

Methods Survey was mailed to 1,830 women and men $\geq 60$ years old in Pennsylvania. Multivariable logistic regression analyses were performed to determine odds ratios for osteoporosis diagnosis and treatment for individuals with established osteoporosis risk factors.

Results Surveys were completed by 1,268 adults (69.3\%). Osteoporosis diagnosis was more commonly reported by participants with risk factors of female sex (OR, 3.60; 95\% CI 2.31-5.61), prolonged oral steroid use (OR, 3.76, 95\% CI 2.06-6.84), low-trauma fracture (OR, 2.14, 95\% CI 1.443.17), height loss (OR, 1.83, 95\% CI 1.28-2.64), and lower weight (OR, 1.35 per $11.4 \mathrm{~kg}$ decrease in weight; $95 \% \mathrm{CI}$,
\end{abstract}

S. Nayak $(\varangle) \cdot$ M. S. Roberts

School of Medicine, Department of Medicine,

Division of General Internal Medicine,

Section of Decision Sciences and Clinical Systems Modeling,

University of Pittsburgh,

Pittsburgh, PA, USA

e-mail: nayaks@upmc.edu

\section{S. L. Greenspan}

School of Medicine, Department of Medicine,

Division of Endocrinology and Metabolism and Division

of Geriatric Medicine, University of Pittsburgh,

Pittsburgh, PA, USA
1.16-1.56). Age and family history of osteoporosis were not predictive of osteoporosis diagnosis, when adjusting for other risk factors. Osteoporosis treatment was more commonly reported by participants with risk factors of female sex (OR, 5.19; 95\% CI, 3.31-8.13), family history (OR, 2.18; 95\% CI, 1.55-3.06), height loss (OR, 1.79; 95\% CI 1.29-2.49), low-trauma fracture (OR, $1.66 ; 95 \% \mathrm{CI}$, 1.14-2.42), and lower weight (OR, 1.45 per $11.4 \mathrm{~kg}$ decrease in weight; $95 \% \mathrm{CI}, 1.27-1.67)$. Osteoporosis treatment was not significantly associated with age or prolonged oral steroid use.

Conclusions Individuals with several established osteoporosis risk factors are more likely to be underdiagnosed or undertreated.

Keywords Diagnosis · Geriatrics · Osteoporosis ·

Survey research

\section{Introduction}

Osteoporosis is common and costly, affecting 10 million women and men in the United States, with direct costs of $\$ 17$ billion in 2005 [1-3]. Approximately one-half of all postmenopausal women and one-quarter of white men over 60 years of age will sustain an osteoporotic fracture in their lifetime $[4,5]$. The 1-year mortality of elderly patients with hip fracture is approximately $24 \%$, and long-term morbidity of osteoporotic fractures can include chronic pain, loss of ability to ambulate, and nursing home placement [6-9].

Although the US Preventive Services Task Force, the National Osteoporosis Foundation, and the American College of Physicians recommend that clinicians screen older adults for osteoporosis [10-12], most individuals with osteoporosis remain undiagnosed and untreated [13-15]. 
The National Ambulatory Medical Care Survey found that fewer than $2 \%$ of women older than 60 years were diagnosed as having osteoporosis by their primary care physicians, even though the expected prevalence in this population is $20 \%$ to $30 \%$; furthermore, appropriate drug therapy was only offered to $36 \%$ of diagnosed patients [15]. Men with osteoporosis appear to be identified and treated even less often than women $[13,14]$.

The objective of our study was to identify patient characteristics associated with diagnosis and treatment of osteoporosis in older adults. We hypothesized that individuals with established osteoporosis risk factors would be more likely to be diagnosed with osteoporosis and receive treatment.

\section{Materials and methods}

Study participants and procedures

We performed a cross-sectional survey of 1,830 women and men age 60 or older, living in or near western Pennsylvania, and enrolled in the University of Pittsburgh's Claude D. Pepper Registry for studies on mobility and balance in older adults. Individuals were recruited for registry participation through mailings to university alumni, faculty, and staff, other ongoing clinical studies at the university, community events at senior citizens centers and a continuing care community, and newspaper advertisements. Nearly all of the registry participants were community dwelling. The study was approved by the University of Pittsburgh Institutional Review Board.

In November 2007, all registry participants were sent a 44-item survey, an informational script describing the purpose of the research study, and a pre-paid, return envelope. Participants were assured that survey responses would remain anonymous and encouraged not to write their names on their returned surveys or return envelope. Payment was not provided for participation. The completed surveys were collected over a 6-month period. Survey data was independently dual-entered into a database by two individuals and validated to ensure integrity.

The survey asked respondents about sociodemographics, osteoporosis risk factors, mobility, falls, prior fractures, prior osteoporosis testing, health beliefs about osteoporosis, and preferences for osteoporosis screening tests. It also asked whether respondents had ever been diagnosed with osteoporosis and whether they had ever taken any medications for osteoporosis other than calcium and vitamin D.

Statistical analyses

We computed descriptive statistics for each survey item. We also performed logistic regression analyses to determine if there were associations between each of the two response variables (diagnosis with osteoporosis and receipt of osteoporosis treatment other than calcium or vitamin D) and the following potential explanatory variables: age (coded in 5-year increments), sex, weight (coded in increments of $11.4 \mathrm{~kg}$ or $25 \mathrm{lb}$ ), self-reported race (white vs black), educational level (completed college vs did not complete college), self-rated health status (poor/fair vs good/very good/excellent), family history of osteoporosis, current smoking, alcohol intake (three or more drinks in one sitting at least four times per week vs less), history of oral steroid use for $>1$ month, height loss $>2.54 \mathrm{~cm}$ (1 in.) over the lifetime, use of arms to get up from a chair most of the time, history of a fall within the past 5 years, and history of a low-trauma fracture (fracture resulting from a fall from standing height or less).

We included individual explanatory variables that showed a significant association with each response variable $(P \leq 0.10)$ as variable candidates in stepwise, backward selection, multivariable logistic regression models. We checked for evidence of interactions between variables and multicollinearity. We considered variables and interaction terms with $P$ values of $\leq 0.05$ to be significant in the final multivariable models. We used Stata version 10.0 (StataCorp, College Station, TX, USA) to perform all analyses.

\section{Results}

Characteristics of survey respondents

Of the 1,830 individuals to whom surveys were sent, 1,268 $(69.3 \%)$ responded (Table 1$)$. Respondents had a mean age of 73.3 years (range, $60-93$; SD, 7.3) and a mean weight of $76.9 \mathrm{~kg}$ (range, 42.6-147.4; SD 16.9). Most respondents were white $(92.9 \%)$, female $(58.7 \%)$, believed that they were in good to excellent health $(88.2 \%)$, and had completed college $(75.0 \%) ; 62.6 \%$ of survey respondents reported being tested for osteoporosis, $22.6 \%$ reported being diagnosed with osteoporosis, and $24.4 \%$ reported osteoporosis treatment other than calcium and vitamin D.

Multivariable models

\section{Diagnosis with osteoporosis}

Respondents were more likely to report osteoporosis diagnosis if they were female (OR, 3.60; 95\% CI $2.31-$ 5.61), had a history of oral steroid use $>1$ month (OR 3.76, 95\% CI 2.06-6.84), had a personal history of low-trauma fracture (OR 2.14, 95\% CI 1.44-3.17), had lost $>2.54 \mathrm{~cm}$ of height over their lifetime (OR 1.83, 95\% CI 1.28-2.64), 
Table 1 Characteristics of the survey respondents

\begin{tabular}{lr}
\hline Characteristics & Number (\%) \\
\hline Sociodemographic characteristics & $664(58.7)$ \\
Female sex & $1,148(92.9)$ \\
White race & $926(75.0)$ \\
Completed college & \\
Osteoporosis-related characteristics & $1,215(96.1)$ \\
$\quad$ Has heard of osteoporosis & $783(62.6)$ \\
Has been screened or tested for osteoporosis & $283(22.6)$ \\
Has been diagnosed with osteoporosis & $307(24.4)$ \\
Has been treated for osteoporosis & \\
$\quad$ (other than calcium/vitamin D) & $236(18.8)$ \\
Has had a low-trauma fracture (fracture resulting & \\
$\quad$ from a fall from standing height or less) & $292(23.8)$ \\
Has a family history of osteoporosis & \\
Other health-related characteristics & $1,114(88.2)$ \\
Has a high self-rated health status & \\
$\quad$ (rated as good, very good, or excellent) & $1,248(98.7)$ \\
Is a nonsmoker & $32(2.6)$ \\
Has a history of alcohol use $\geq 4$ times per & \\
$\quad$ week, $\geq 3$ drinks at a time & $103(8.2)$ \\
Has a history of oral steroid use for more than 1 month & $152(91.3)$ \\
Has experienced a height loss $>2.54$ cm & $435(35.3)$ \\
$\quad$ ( 1 in.) over the lifetime & \\
Uses arms to get up from a chair most of the time & $460(36.8)$ \\
Has fallen within the past 5 years & \\
Is ambulatory without the use of an assistive device & 1,1509 \\
\hline
\end{tabular}

There were 1,268 survey respondents. However, there were missing data for each of the characteristics listed in this table. The percentage of missing data for sex was $10.8 \%$, but percentages of missing data for other characteristics were below $4 \%$. The percentages shown here reflect the percentages of individuals who responded to the question about the characteristic listed. Mean age of respondents was 73.3 years (range, 60-93; SD, 7.3). Mean weight was $76.9 \mathrm{~kg}$ (range, 42.6147.4; SD 16.9)

or had a lower weight (OR, 1.35 per $11.4 \mathrm{~kg}$ decrease in weight; 95\% CI, 1.16-1.56). There was a significant positive interaction between age and family history of osteoporosis (OR 1.44; 95\% CI 1.11-1.86) and a significant negative interaction between family history of osteoporosis and oral steroid use $>1$ month (OR 0.26, 95\% CI 0.07-0.88). When we included these interactions in the model, age and family history of osteoporosis by themselves were not significant predictors of osteoporosis diagnosis. There was no evidence of multicollinearity in this model. Osteoporosis diagnosis was not significantly associated with race, alcohol intake, smoking status, educational level, self-rated health status, use of arms to get up from a chair, or history of a fall within the past 5 years.

\section{Receipt of osteoporosis treatment}

Respondents were more likely to report osteoporosis treatment if they were female (OR, 5.19; 95\% CI, 3.31-8.13), had a family history of osteoporosis (OR, 2.18; 95\% CI, 1.55 3.06 ), had lost $>2.54 \mathrm{~cm}$ of height over their lifetime (OR, $1.79 ; 95 \%$ CI $1.29-2.49$ ), had a history of low-trauma fracture (OR, 1.66; 95\% CI, 1.14-2.42), or had a lower weight (OR, 1.45 per $11.4 \mathrm{~kg}$ decrease in weight; $95 \% \mathrm{CI}, 1.27-1.67$ ). There was no evidence of multicollinearity or significant interactions between the variables included in this model. Receipt of osteoporosis treatment was not significantly associated with age, history of oral steroid use for $>1$ month, race, alcohol intake, smoking status, educational level, selfrated health status, use of arms to get up from a chair, or history of a fall within the past 5 years.

\section{Discussion}

Our survey of 1,268 women and men aged 60 and older suggests that individuals with several established osteoporosis risk factors may be underdiagnosed and undertreated. Most notably, older respondents were no more likely than younger respondents to be diagnosed with osteoporosis or receive treatment other than calcium and vitamin $\mathrm{D}$, when adjusting for other osteoporosis risk factors. This finding is remarkable because age is the strongest individual risk factor for osteoporosis, with older individuals having the highest prevalences of osteoporosis in epidemiological studies $[16,17]$. Other surprising findings included that individuals with several other established osteoporosis risk factors - such as family history, prolonged oral steroid use, white race, smoking, and heavy alcohol consumption - were either no more likely to be diagnosed with osteoporosis or no more likely to be treated for osteoporosis, after adjusting for other risk factors. However, we did find that individuals with osteoporosis risk factors of female sex, lower body weight, height loss, and history of low-trauma fracture were more likely to be diagnosed and treated than individuals without these risk factors. Thus, our results were mixed with respect to our hypothesis that individuals with established osteoporosis risk factors would be more likely to be diagnosed with osteoporosis and receive treatment.

Several of our findings are consistent with results of earlier studies. Multiple previous studies suggest that older individuals are either less likely or no more likely than younger individuals to be treated for osteoporosis [18-21]. A few studies have found that younger patients are less likely to receive pharmacologic treatment for osteoporosis than older patients, but this discrepancy may be secondary to the use of younger age cutoffs to distinguish older from younger patients in these particular studies (e.g., postmenopausal vs premenopausal) [22-24]; our study focused on an older population of individuals, those age 60 and older. Our finding that individuals with prolonged oral steroid use may not be receiving sufficient osteoporosis treatment 
concurs with that of other studies $[22,25,26]$, as does our finding that osteoporosis treatment was more likely in women than men [18, 21-23]. We also observed that osteoporosis treatment was no more likely in white adults than black adults, when adjusting for other osteoporosis risk factors; this finding is different from that of previous studies and warrants further study [18].

Our findings further advance the understanding of current patterns of osteoporosis diagnosis and treatment by suggesting that individuals with particular osteoporosis risk factors may be overlooked for diagnosis and treatment. Most significant is the observation that older individuals are not more likely to be diagnosed and treated than younger individuals. Older individuals are at highest risk for osteoporotic fractures, particularly hip fracture, which is associated with significant morbidity, mortality, and costs. If older adults are underdiagnosed and undertreated, this represents an important opportunity to change clinical practice to improve osteoporosis outcomes. Likewise, our results also suggest that individuals with other risk factors, such as prolonged oral steroid treatment and family history of osteoporosis, may need to be better targeted for osteoporosis identification and treatment to improve outcomes.

To our knowledge, our study is the largest patient survey of characteristics associated with osteoporosis diagnosis and treatment. However, the study had several limitations. First, because the survey was based on self-report, there may have been recall bias concerning osteoporosis diagnosis and treatment. Second, the survey population consisted of individuals who lived in or near western Pennsylvania, volunteered for a research registry, and were disproportionately white, healthy, and highly educated, which may limit the generalizability of our results. However, it is possible that if even in this survey population individuals with several known risk factors for osteoporosis were not more likely to receive osteoporosis diagnosis or treatment, this may be an even larger problem in the general population of older adults. Third, our study had small numbers of individuals with certain osteoporosis risk factors, such as smokers and heavy alcohol drinkers, which may have limited our ability to detect an association between these characteristics and osteoporosis diagnosis or treatment. Our study also had several notable strengths, including a large sample size, nearly $70 \%$ response rate, and inclusion of both female and male participants.

In conclusion, we found that individuals with several key osteoporosis risk factors, such as advanced age, prolonged oral steroid use, and family history of osteoporosis, were either not more likely to receive osteoporosis diagnosis or not more likely to obtain treatment, when adjusting for other osteoporosis risk factors. Our results suggest that individuals with these risk factors are more likely to be underdiagnosed or undertreated. Future investigations should confirm our findings in other study populations and investigate interventions to improve osteoporosis diagnosis and treatment rates in individuals at highest risk.

Acknowledgements The authors thank Anna K. Ercius, MPH, for mailing surveys, data collection, and data entry; Deljo Gannon for data entry and validation; Linda Quinn and Terry Sefcik, MSIS, for assistance with survey design; the University of Pittsburgh Claude D. Pepper Older Americans Independence Center for access to a registry of individuals interested in research participation; and all of the individuals who responded to our survey.

Funding This study was supported by grants KL2 RR024154-02 and UL1 RR024153 from the National Center for Research Resources (NCRR), a component of the National Institutes of Health (NIH) and NIH Roadmap for Medical Research (Dr. Nayak); grant K24 DK062895 from the National Institute of Diabetes and Digestive and Kidney Diseases (Dr. Greenspan); and grant P30 AG024827 from the National Institute on Aging (University of Pittsburgh Claude D. Pepper Older Americans Independence Center). The contents of this manuscript are solely the responsibility of the authors and do not necessarily represent the official view of the NCRR or NIH.

Conflicts of interest None.

Open Access This article is distributed under the terms of the Creative Commons Attribution Noncommercial License which permits any noncommercial use, distribution, and reproduction in any medium, provided the original author(s) and source are credited.

\section{References}

1. U.S. Department of Health and Human Services (2004) Bone health and osteoporosis: a report of the surgeon general. Rockville, MD: U.S. Department of Health and Human Services, Office of the Surgeon General

2. Looker AC, Orwoll ES, Johnston CC Jr et al (1997) Prevalence of low femoral bone density in older U.S. adults from NHANES III. J Bone Miner Res 12:1761-1768

3. Burge R, Dawson-Hughes B, Solomon DH et al (2007) Incidence and economic burden of osteoporosis-related fractures in the United States, 2005-2025. J Bone Miner Res 22:465-475

4. Nelson HD, Helfand M, Woolf SH et al (2002) Screening for postmenopausal osteoporosis: a review of the evidence for the U. S. Preventive Services Task Force. Ann Intern Med 137:529-541

5. Nguyen TV, Eisman JA, Kelly PJ et al (1996) Risk factors for osteoporotic fractures in elderly men. Am J Epidemiol 144:255-263

6. Huddleston JM, Whitford KJ (2001) Medical care of elderly patients with hip fractures. Mayo Clin Proc 76:295-298

7. Braithwaite RS, Col NF, Wong JB (2003) Estimating hip fracture morbidity, mortality and costs. J Am Geriatr Soc 51:364-370

8. Nevitt MC, Ettinger B, Black DM et al (1998) The association of radiographically detected vertebral fractures with back pain and function: a prospective study. Ann Intern Med 128:793-800

9. Oleksik A, Lips P, Dawson A et al (2000) Health-related quality of life in postmenopausal women with low BMD with or without prevalent vertebral fractures. J Bone Miner Res 15:1384-1392

10. U.S. Preventive Services Task Force (2002) Screening for osteoporosis in postmenopausal women: recommendations and rationale. Ann Intern Med 137:526-528 
11. The National Osteoporosis Foundation (NOF) (2008) Clinician's guide to prevention and treatment of osteoporosis. National Osteoporosis Foundation, Washington, DC

12. Qaseem A, Snow V, Shekelle P et al (2008) Screening for osteoporosis in men: a clinical practice guideline from the American College of Physicians. Ann Intern Med 148:680-684

13. Kiebzak GM, Beinart GA, Perser K et al (2002) Undertreatment of osteoporosis in men with hip fracture. Arch Intern Med 162:2217-2222

14. Morris CA, Cabral D, Cheng $\mathrm{H}$ et al (2004) Patterns of bone mineral density testing: current guidelines, testing rates, and interventions. J Gen Intern Med 19:783-790

15. Gehlbach SH, Fournier M, Bigelow C (2002) Recognition of osteoporosis by primary care physicians. Am J Public Health 92:271-273

16. Riggs BL, Melton LJ 3rd (1995) The worldwide problem of osteoporosis: insights afforded by epidemiology. Bone 17:505S-511S

17. Siris ES, Miller PD, Barrett-Connor E et al (2001) Identification and fracture outcomes of undiagnosed low bone mineral density in postmenopausal women: results from the National Osteoporosis Risk Assessment. JAMA 286:2815-2822

18. Solomon DH, Brookhart MA, Gandhi TK et al (2004) Adherence with osteoporosis practice guidelines: a multilevel analysis of patient, physician, and practice setting characteristics. Am J Med 117:919-924
19. Kirk JK, Spangler JG, Celestino FS (2000) Prevalence of osteoporosis risk factors and treatment among women aged 50 years and older. Pharmacotherapy 20:405-409

20. Freedman KB, Kaplan FS, Bilker WB et al (2000) Treatment of osteoporosis: are physicians missing an opportunity? J Bone Joint Surg Am 82-A:1063-1070

21. Yood RA, Harrold LR, Fish L et al (2001) Prevention of glucocorticoid-induced osteoporosis: experience in a managed care setting. Arch Intern Med 161:1322-1327

22. Solomon DH, Katz JN, Jacobs JP et al (2002) Management of glucocorticoid-induced osteoporosis in patients with rheumatoid arthritis: rates and predictors of care in an academic rheumatology practice. Arthritis Rheum 46:3136-3142

23. Mudano A, Allison J, Hill J et al (2001) Variations in glucocorticoid induced osteoporosis prevention in a managed care cohort. J Rheumatol 28:1298-1305

24. Morris CA, Cheng H, Cabral D et al (2004) Predictors of screening and treatment of osteoporosis: a structural review of the literature. Endocrinologist 14:70-75

25. Curtis JR, Westfall AO, Allison JJ et al (2005) Longitudinal patterns in the prevention of osteoporosis in glucocorticoid-treated patients. Arthritis Rheum 52:2485-2494

26. Shah SK, Gecys GT (2006) Prednisone-induced osteoporosis: an overlooked and undertreated adverse effect. J Am Osteopath Assoc 106:653-657 